\title{
A comparative study of MRI versus arthroscopic findings in meniscal injuries of knee
}

\author{
Baghel A.S. ${ }^{1}$, Singhania K.V. ${ }^{2}$ \\ ${ }^{1}$ Dr. Arvindra Singh Baghel, Senior Resident, ${ }^{2}$ Dr. Khushboo Vinod Singhania, Senior Resident; both authors are \\ attached with Department of Radiology, AIIMS, Bhopal, Madhya Pradesh, India.
}

Corresponding Author: Dr. Khushboo Vinod Singhania

\begin{abstract}
Introduction: Knee joint is one of the most commonly injured joints because of its anatomical structure, its exposures to external forces and the functional demands placed on it. Because of the difficulty of obtaining an accurate clinical examination in the acute setting, the incidence of such injuries is not well understood. Material and methods: The present study was carried out in 30 patients having clinical signs and symptoms of knee injury over a period of 12 months, who were referred to the radiological department from different IPD's and OPD's of Himalayan Institute of Medical Sciences, Swami Ram Nagar, Dehradun. Results: Diagnostic accuracy of MRI was 86.6\% for medial meniscus and $93 \%$ for lateral meniscus in our study. In present study medial meniscal injuries was found in 16 patients (53.3\%). Lateral meniscal injuries were found in 8 patients (26.6\%). Conclusion: In conclusion, the present study supports that MRI is helpful in diagnosing meniscal injuries. Now days patients expectations are maximal and taking into account that MRI false or misleading results can be as high as 20-30 percent in specific knee pathologies. Undoubtly new techniques and more powerful tomograms will improve MRI's accuracy leading to better diagnostic accuracy in knee injuries.
\end{abstract}

Keywords: Accuracy, Sensitivity, Specificity, Meniscal injuries

\section{Introduction}

Knee joint is one of the most commonly injured joints because of its anatomical structure, its exposures to external forces and the functional demands placed on it. Because of the difficulty of obtaining an accurate clinical examination in the acute setting, the incidence of such injuries is not well understood $[1,2]$.

Recently, new diagnostic protocols have been developed to improve the diagnosis of knee injuries with high-resolution magnetic resonance imaging (MRI) scans [3-5]. These new imaging protocols have been shown to be both sensitive and specific for most structures [3].

Diagnostic arthroscopy is an important advance, improving diagnostic accuracy from 64 to 94 per cent. However it is an invasive procedure, with the possible attendant complications of infection, hemarthrosis, adhesions, reflex sympathetic dystrophy [6]. Magnetic Resonance Imaging (MRI) scanning of the knee joint has often been regarded as the non-invasive alternative

Manuscript received: $5^{\text {th }}$ February 2018

Reviewed: $15^{\text {th }}$ February 2018

Author Corrected: $20^{\text {th }}$ February 2018

Accepted for Publication: 24 ${ }^{\text {th }}$ February 2018 to diagnostic arthroscopy. MRI scan has now been routinely used to support the diagnosis for meniscal or cruciate ligament injuries prior to recommending arthroscopic examination and surgery [7].

The comparison of MRI diagnosis and surgical/clinical findings has always been a challenge for the health professions. Review of the available literature suggests that there are a number of studies looking at these diagnostic toolsand only limited studies are available taking them together [8].

So our study is designed to compare and correlate these methods in the diagnosis of internal derangement of knee.

\section{Material and Methods}

\section{Study Design}

- Type of the study: An observational and descriptive study.

- Sample size and Sampling method: A minimum number of 30 patients. 


\section{Original Research Article}

\section{Selection of Subject}

Inclusion criteria- All patients with history suggestive of traumatic internal derangement of knee who underwent both MRI and arthroscopy

\section{Exclusion criteria}

- Patients having major knee trauma other than internal derangement of knee.

- Patients having non traumatic pathologies in the knee.

- History of previous knee operation

Study Tools: Structured Study instruments (case reporting form) was developed and used to generate data. All patients with clinical suspicion of internal derangement of the knee were subjected to MR examination followed by arthroscopy.

MRI was performed on Machine 1.5 Tesla MR Unit: AVANTO, SIEMENS (Germany) using dedicated knee coil for optimal signal acquisition.

Ethical committee clearance- Institutional ethical committee permission was taken before conduction of the study and informed consent was taken from all the participants included in the study.

Statistical Analysis- The statistical analysis was done by using SPSS software.Sensitivity, specificity, positive predictive value and negative predictive value was calculated.

\section{Results}

The present study was carried out in 30 patients having clinical signs and symptoms of knee injury over a period of 12 months, who were referred to the radiological department from different IPD's and OPD's of Himalayan Institute of Medical Sciences, Swami Ram Nagar, Dehradun.

All the 30 subjects underwent a dedicated MR knee examination as per the protocol for detailed evaluation of the internal derangements of the injured knee. MR knee findings were recorded as per the documentation chart in the proforma. Intraoperative arthroscopic surgical findings were recorded in those patients who had undergone arthroscopy. The following observations were noted in the study:

Table-1: Comparison of MRI and arthroscopy findings in meniscal injury.

\begin{tabular}{|l|c|c|c|c|}
\hline \multicolumn{2}{|c|}{ Medial Meniscus } & \multicolumn{3}{|c|}{ Arthroscopy } \\
\cline { 3 - 5 } & Tear & $14(\mathrm{TP})$ & No Tear & Total \\
\hline \multirow{3}{*}{ MR Examination } & No Tear & $2(\mathrm{FN})$ & $12(\mathrm{TN})$ & 16 \\
\cline { 2 - 5 } & Total & 16 & 14 & 30 \\
\cline { 2 - 5 } & &
\end{tabular}

- Sensitivity $=\mathrm{TP} /(\mathrm{TP}+\mathrm{FN})=87.50 \%$

- Specificity $=\mathrm{TN} /(\mathrm{FP}+\mathrm{TN})=85.71 \%$

- $\mathrm{PPV}=\mathrm{TP} /(\mathrm{TP}+\mathrm{FP})=87.50 \%$

- $\mathrm{NPV}=\mathrm{TN} /(\mathrm{TN}+\mathrm{FN})=85.71 \%$

- Accuracy $=(\mathrm{TP}+\mathrm{TN}) /(\mathrm{TP}+\mathrm{TN}+\mathrm{FP}+\mathrm{FN})=86.66 \%$

Table-2: Comparison of MRI and arthroscopy findings in meniscal injury

\begin{tabular}{|c|c|c|c|c|}
\hline \multicolumn{2}{|c|}{ Lateral Meniscus } & \multicolumn{3}{c|}{ Arthroscopy } \\
\hline \multirow{2}{*}{ MR Examination } & Tear & $8(\mathrm{TP})$ & No Tear & 0 \\
\hline & No Tear & $2(\mathrm{FN})$ & $20(\mathrm{TN})$ & 22 \\
\hline & Total & 10 & 20 & 30 \\
\hline
\end{tabular}

- Sensitivity $=\mathrm{TP} /(\mathrm{TP}+\mathrm{FN})=80.00 \%$

- Specificity $=\mathrm{TN} /(\mathrm{FP}+\mathrm{TN})=100.00 \%$

- $\mathrm{PPV}=\mathrm{TP} /(\mathrm{TP}+\mathrm{FP})=100.00 \%$

- $\mathrm{NPV}=\mathrm{TN} /(\mathrm{TN}+\mathrm{FN})=90.91 \%$

- Accuracy $=(\mathrm{TP}+\mathrm{TN}) /(\mathrm{TP}+\mathrm{TN}+\mathrm{FP}+\mathrm{FN})=93.3 \%$ 
Original Research Article

\section{Discussion}

Our study of 30 patients with knee injury was referred to us from various OPD's and IPD's and the study was conducted in the Department of Radiodiagnosis, Himalayan Institute of Medical Sciences, Swami Rama Nagar, Dehradun. The MR findings were correlated with the arthroscopic findings.

In present study medial meniscal injuries was found in 16 patients $(53.3 \%)$ with $43.7 \%$ cases showing grade 3 injury. Lateral meniscal injuries were found in 8 patients $(26.6 \%)$ with $37.5 \%$ cases showing grade 3 injury. Grade III tears and horizontal tear was more common in both the menisci. There is preponderance of MM tears over LM tears in our study which is well correlated with the study done by Singh JP et al [9] in a series of 173 cases of which they found 57 (38.23\%) patients showed MM tear and 28 (29.41\%) patients showed LM tear. Out of 173 patients, Grade 3 tear of MM was seen in 57 (32.95\%) patients.

In our study, we found 15 cases of combined injuries and the most common combination was found to be ACL-MM injury seen in $40 \%$ of the cases followed by ACL-LM and PCL-LM accounting for $13.66 \%$ each. This was in concordance with the study by Esmaili Jah AA et al [10], which showed 17 cases of concomitant injuries on MRI with the commonest combination of anterior cruciate ligament rupture and medial meniscus tear $(38 \%)$.

Diagnostic accuracy of MRI was $86.6 \%$ for medial meniscus and $93 \%$ for lateral meniscus in our study which corresponds to studies done by Noha H. Behairy at al [11] 73\% for MM and $78.5 \%$ for LM, Glashow et al [12] $74 \%$ for MM and $94 \%$ for LM, Rappeport et al [13] 77\% for MM and 91\% for LM, Kinnunen et al [14] $82 \%$ for MM and $88 \%$ for LM and Incesu et al [15] $86 \%$ accuracy rate for menisci.

In our study, the sensitivity and specificity of MRI in diagnosing medial meniscus injury was $87.5 \%$ and $85.71 \%$ respectively whereas the sensitivity and specificity of MRI in diagnosing lateral meniscus injury was $80 \%$ and $100 \%$ respectively. In a study conducted by Kumar S, Bansal A et al for Meniscal injuries the sensitivity was $94 \%$, specificity was $94.5 \%$ by MRI, sensitivity was $100 \%$ and specificity was $100 \%$ for Arthroscopy [16].

The role of magnetic resonance imaging has steadily increased and now it has become the first line investigation for most of the lesions of knee. It is also being used for pre and postoperative evaluation. Complete evaluation of all the internal structures of the knee was not possible with other modalities like conventional radiography, arthrography, ultrasonography and computed tomography. Even with arthroscopy lesions such as peripheral meniscal tears, inferior surface tears and osteochondritis dessicans without articular cartilage damage are most often not detected. Multiplanar MR images provide significant improvement in assessing these structures.

\section{Conclusion}

In conclusion, the present study supports that MRI is helpful in diagnosing meniscal injuries. Now a days patients' expectations are maximal and taking into account that MRI false or misleading results can be as high as 20-30 percent in specific knee pathologies, it is concluded that arthroscopy still remains the gold standard in diagnosing the internal knee lesions. Undoubtly new techniques and more powerful tomograms will improve MRI's accuracy leading to better diagnostic accuracy in knee injuries.

MRI is unique in its ability to evaluate structure as well as the surface of the meniscus. MRI is also able to evaluate the structures not accessible on arthroscopy like evaluation of bone and collateral ligaments.

\section{Funding: Nil, Conflict of interest: None Permission of IRB: Yes}

\section{References}

1. Hughston JC, Andrews JR, Cross MJ, Moschi A. Classification of knee ligament in stabilities. Part II. The lateral compartment. J Bone Joint Surg Am 1976; 58: 173-179.

2. Hughston JC, Jacobson KE. Chronic posterolateral rotatory instability of the knee. J Bone Joint Surg Am 1985;67:351-359.

3. La Prade RF, Gilbert TJ, Bollom TS, Wentorf F, Chaljub G.The magnetic resonance imaging appearance of individual structures of the posterolateral knee. A prospective study of normal knees and knees with surgically verified grade III injuries. Am J Sports Med 2000; 28:191-199.

4. Miller TT, Gladden P, Staron RB, Henry JH, Feldman F. Posterolateral stabilizers of the knee: 
Original Research Article

Anatomy and injuries assessed with MR imaging. AJR Am J Roentgenol 1997;169: 1641-1647.

5. Ruiz ME, Erickson SJ. Medial and lateral supporting structures of the knee. Normal MR imaging anatomy and pathologic findings. Magn Reson Imaging Clin N Am 1994;2:381- 399.

6. Haven D and Collins HR. Diagnosis of internal derangement of the knee: The role of Arthrography. J Bone and Joint Surg.1975; 57: 802-10.

7. Munshi M, Davidson M, MacDonald PB, Froese W, Sutherland K. The efficacy of magnetic resonance imaging in acute knee injuries. Clin J Sport Med.2000; $10: 34-9$.

8. Mudhusudan TR, Kumar TM, Bastawrous SS, Sinha A. Clinical examination, MRI and arthroscopy in meniscal and ligamental knee injuries- a prospective study. Journal of Orthopaedic Surgery and Research. 2008; 15: 3-19.

9. Singh JP, Garg L, Shrimali R, Setia V, Gupta V. MR Imaging of knee with arthroscopic correlation in twisting injuries. IJRI. 2004;14: 33-40.

10. EsmailiJah AA, Sohrab K, Zarei R, Moghaddam AK. Accuracy of MRI in comparison with Clinical and Arthroscopic findings in ligamentous and meniscal injuries of the knee.Acta Orthop Belg. 2005; 71:189-96.
11. Noha H. Behairy, Mohsen A. Dorgham, Sherif. A.K. Accuracy of routine MRI in meniscal and ligamentous injuries of the knee: comparision with arthroscopy. Int Orthop SICOT. 2009;33(4): 961-7.

12. Glashow JL, Katz R, Schnieder M, Scott W. Double-blind assessment of the value of magnetic resonance imaging in the diagnosis of anterior cruciate and meniscal lesions. J Bone Joint Surg Am. 1989; 71: 113-9.

13. Reppeport ED, Mehta S, Wieslander SB, Lausten GS, Thomsen HS. MR imaging before arthroscopy in knee joint disorders. Acta Radiol. 1996;37(5): 602-9.

14. Kinnunen J, Bondestam S, K ivioja A, Ahovuo J, Toivakka SK, T ulikoura I et al. Diagnostic performance of low field MRI in acute knee injuries. Magn Reson Imaging. 1994; 12: 1155-60.

15. Incesu L, Dabak N, BeletU, Mazhar EL, Gulman B. Comparision of MRI and arthroscopic findings in knee Joint pathologies. Turk J Med Res. 1997; 15 (1): 21-5.

16. Kumar S, Bansal A. A Comparative Study of Accuracy of Clinical Examination, MRI \& Arthroscopy Findings in Cruciate Ligament and Meniscal Injuries of the Knee. Indian Journal of Basic \& Applied Medical Research; December 2011: Issue-1, Vol.-1, P. 106-113

\section{How to cite this article?}

Baghel A.S, Singhania K.V. A comparative study of MRI versus arthroscopic findings in meniscal injuries of knee. Int $J$ Med Res Rev 2018;6 (03):192-195. doi:10.17511/ijmrr. 2018.i03.10. 